\title{
A novel digital beamformer applied in vehicle mounted HF receiving device
}

\author{
Huajun Zhang, Huotao Gao ${ }^{\text {a) }}$, Qingchen Zhou, Lin Zhou, \\ and Fan Wang
}

Electronic Information Department, Wuhan University, Luoyu Road No.129, Wuhan, 430072, China

a)gaoght863@163.com

\begin{abstract}
In this work, a novel digital beamformer applied in vehicle-mounted high frequency $(\mathrm{HF})$ receiving device has been developed. The system adopts robust superdirective beamforming algorithm, which not only effectively reduces the receiving array aperture but also maintains a higher directive gain compared with conventional beamforming method (CBF). The system is designed based on the idea of radio-defined software (SDR), which is easy to update. It contains 16 receiving channels and can output 3 analog beam signals. Experiment shows that the system has fast response and is feasible in engineering.
\end{abstract} Keywords: digital beamformer, robust superdirective beamforming, uniform circular array, software-defined radio

Classification: Microwave and millimeter wave devices, circuits, and systems

\section{References}

[1] S. Hong, D. Yang, K. Liu, X. He and M. M. Tentzeris: IEICE Electron. Express 6 [16] (2009) 1192.

[2] S. A. Schelkunoff: Bell Syst. Tech. J. 22 [1] (1943) 80.

[3] Y. L. Ma, Y. X. Yang, Z. Y. He, et al: IEEE Trans. Ind. Electron. 60 [6] (1996) 67.

[4] Q. Zhou, H. Gao, H. Zhang and F. Wang: Progress In Electromagnetics Research. 136 (2013) 665.

[5] M. Uzsoky and L. Solymar: IEEE Trans. Antennas Propag. 60 [2] (2013) 203.

[6] L. Zhou, F. Wang, H. Gao, Y. Sun and H. Zhang: IEICE Electron. Express 9 [13] (2013) 1102.

[7] J. Li, P. Stoica and Z. S. Wang: IEEE Trans. Signal Process. 52 [9] (2004) 2407.

\section{Introduction}

(c) IEICE 2014

DOI: 10.1587/elex.11.20130919 Received November 22, 2013 Accepted December 20, 2013 Publicized January 10, 2014

Copyedited January 25, 2014
High frequency (HF) signal is characterized by long-distance propagation without relay network and has been widely applied in radar, remote sensing 
and communication field. But at the same time, there exist some common disadvantages such as energy attenuation and multi-path effects due to large amount of reflection and refraction during spreading. In addition, HF band is crowded and has serious co-channel interference. Digital beamforming (DBF) has a good suppression on multi-path fading and co-channel interference. However, for obtaining the best direction gain, most of the existing methods require that the array element spacing is half-wave length [1]. This will need huge array aperture in HF system, which brings a lot of inconvenience in system setting up and maintenance. Therefore, beamformer using conventional antenna array is unrealistic in vehicle-mounted receiving device.

In order to adapt the characteristics of vehicle equipment, compactly spaced antenna array has to be applied. However, the directive gain of conventional beamforming method is unsatisfactory in this mode, especially at the low frequency end of HF. In order to achieve better performance with mini array, researchers have proposed the concept of superdirective array synthesis since 1940s [2]. Likewise, because of its high sensitivity to array error, the actual performance has a great gap with theoretical simulation. For improving algorithm robustness, many works have been done. To sum up, diagonal loading method is the most representative [3]. The defect of this method is the difficulty in choosing a proper loading value, which can't make a good trade-off between robustness and directive gain. Therefore, it is not practical in engineering.

The authors propose a robustness superdirective beamforming algorithm [4]. This method uses a parameter called sensitivity factor. By choosing different $K$ value, people can easily make a trade-off between robustness and directive gain according to the actual array error. Further, a digital beamformer (DBF) system based on this algorithm is designed. The system uses the concept of software-defined radio (SDR), easy to update software in different electromagnetic environment. The DBF system can achieve weighted summation of up to 16 channels and output multiple beams at the same time with digital upconverters (DUCs). The experiment results proves its feasibility and better performance than conventional beamforming.

\section{Robust superdirective beamforming algorithm}

For a uniform circular array with $M$ antenna elements, we assume the weight vector as $\mathbf{w}$ and the received snapshot data is $X(t)=\left[x_{1}(t), x_{2}(t), \cdots, x_{M}(t)\right]^{T}$. The summed output can be written as:

$$
Y(t)=\mathbf{w}^{H} X(t)
$$

For conventional beamforming $(\mathrm{CBF})$, the weight vector is $\mathbf{w}=\mathbf{a}\left(\theta_{m}, \phi_{m}\right)$, where $\mathbf{a}\left(\theta_{m}, \phi_{m}\right)$ represents the array steer vector. By adjusting the azimuth angle $\theta_{m}$ and the pitch angle $\phi_{m}$ of steer vector, beam pattern can point to any direction. Compared with $\mathrm{CBF}$, the weight vector computation of superdirective beamforming is relatively more complexed. Correspondingly, its performance is better than $\mathrm{CBF}$, which can be proved by comparative 
verification in section 4 . The sensitivity factor [5] is defined as:

$$
K=\frac{\mathbf{w}^{H} \mathbf{w}}{\mathbf{w}^{H} N \mathbf{w}}
$$

in which $N=\mathbf{a}\left(\theta_{m}, \phi_{m}\right) \mathbf{a}^{H}\left(\theta_{m}, \phi_{m}\right)$. The denominator of Equation (2) represents the power of main lobe direction. Smaller $K$ is, the array is more robust. The directive gain of array is represented as:

$$
G\left(\theta_{m}, \phi_{m}\right)=\frac{\mathbf{w}^{H} N \mathbf{w}}{\mathbf{w}^{H} R \mathbf{w}}
$$

where $R=\frac{1}{4 \pi} \int_{0}^{2 \pi} \int_{0}^{\pi} \sin \theta \mathbf{a}(\theta, \varphi) \mathbf{a}^{H}(\theta, \varphi) d \theta d \varphi$. When the array element adopts short vertical dipole, the $R$ matrix can be simplified as:

$$
R_{i j}= \begin{cases}\frac{2}{3}, & i=j \\ \frac{k d_{i j}}{\sin k d_{i j}}-\frac{1}{\left(k d_{i j}\right)^{2}}\left[\frac{k d_{i j}}{\sin k d_{i j}}-\cos k d_{i j}\right], & i \neq j .\end{cases}
$$

where $k$ is the radio wave number and $d_{i j}$ is the distance between the $i$ th element and the $j$ th element. In order to ensure the response of main lobe direction is distortionless, we have to make $\mathbf{w}^{H} \mathbf{a}\left(\theta_{m}, \phi_{m}\right)=1$. Thus, Equation (2) and (3) can be further simplified as:

$$
K=\mathbf{w}^{H} \mathbf{w}, \quad G\left(\theta_{m}, \phi_{m}\right)=\frac{1}{\mathbf{w}^{H} R \mathbf{w}}
$$

For maximum directive gain $G\left(\theta_{m}, \phi_{m}\right)$, we just need to minimize the denominator. Thus, under the condition of given sensitivity factor $K$, the constraints above can be rewritten as :

$$
\left\{\begin{array}{l}
\min _{\mathbf{w}} \mathbf{w}^{H} R \mathbf{w} \\
\mathbf{w}^{H} \mathbf{a}\left(\theta_{m}, \phi_{m}\right)=1 \\
\|\mathbf{w}\|=K
\end{array}\right.
$$

Actually, this method makes a tradeoff between robustness and directive gain by selecting proper $K$ value. If array error is small, we can select a larger $K$ value, which sacrifices some robustness to improve directive gain. If array error is large, a smaller $K$ value can be adopted, which sacrifices some directive gain to guarantee robustness. The detailed description about how to choose a proper $K$ value is introduced in paper [4]. Generally, 0.2-0.7 is a relatively proper interval if the actual amplitude error is below $9 \mathrm{~dB}$ and the actual phase error is below 10 degree. To solve the constraints (6) for $\mathbf{w}$, Lagrange method is adopted. The target function can be constructed as:

$$
L=\mathbf{w}^{H} R \mathbf{w}+\hat{\lambda}\left(\mathbf{w}^{H} \mathbf{w}-K\right)+\mu\left(\mathbf{w}^{H} \mathbf{a}\left(\theta_{m}, \phi_{m}\right)-1\right)
$$

where $\hat{\lambda}$ and $\mu$ are the lagrange multipliers. Taking its gradient with respect to $\mathbf{w}$ and making $\nabla_{\mathbf{w}}(L)=0$, we get:

$$
\mathbf{w}=\mu(R+\hat{\lambda} I)^{-1} \mathbf{a}\left(\theta_{m}, \phi_{m}\right)
$$

where $I$ is the unit matrix. Inserting (8) into the equation $\mathbf{w}^{H} \mathbf{a}\left(\theta_{m}, \phi_{m}\right)=1$ leads to:

$$
\mu=\left[\mathbf{a}^{H}\left(\theta_{m}, \phi_{m}\right)(R+\hat{\lambda} I)^{-1} \mathbf{a}\left(\theta_{m}, \phi_{m}\right)\right]^{-1}
$$


Thus, the Equation (8) can be rewritten as:

$$
\mathbf{w}=\frac{(R+\hat{\lambda} I)^{-1} \mathbf{a}\left(\theta_{m}, \varphi_{m}\right)}{\mathbf{a}^{H}\left(\theta_{m}, \varphi_{m}\right)(R+\hat{\lambda} I)^{-1} \mathbf{a}\left(\theta_{m}, \varphi_{m}\right)}
$$

Equation (10) is the final formula of weight vector computation. For lagrange multiplier $\hat{\lambda}$, we can calculate it by numerical method, which will be introduced in section 3.3.

\section{System design and implementation}

The architecture of the DBF system is showed in Fig. 1. Analog signals are digitizied by analog-to-digital converter (ADC) chips firstly. Then it completes digital signals extraction and filtering in digital downconverters

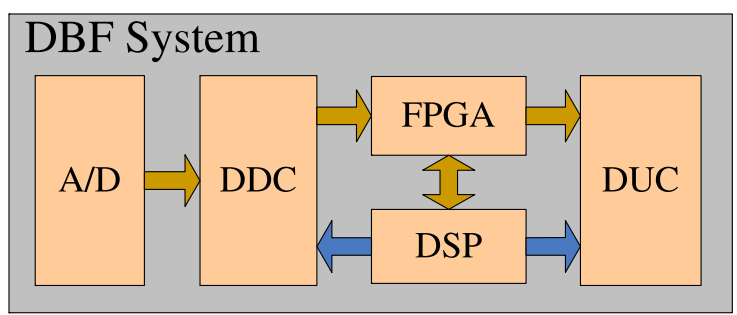

Fig. 1. Hardware Architecture of DBF.

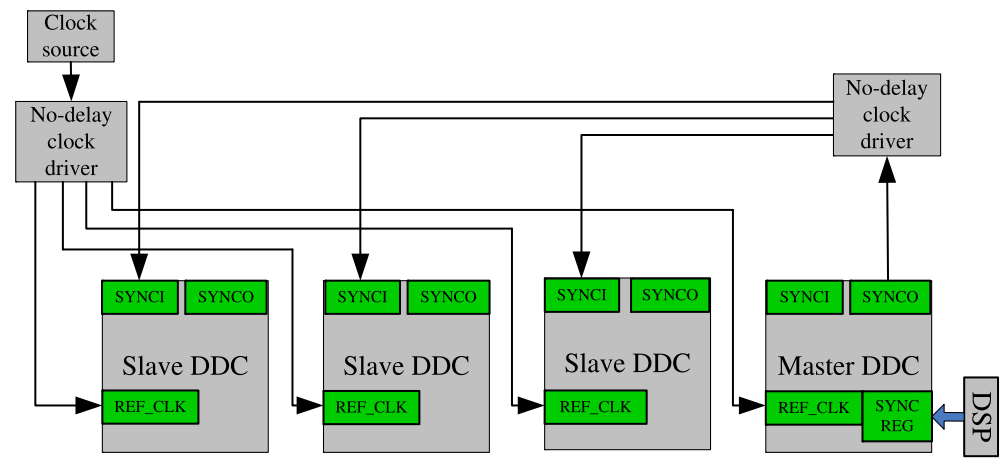

(a)

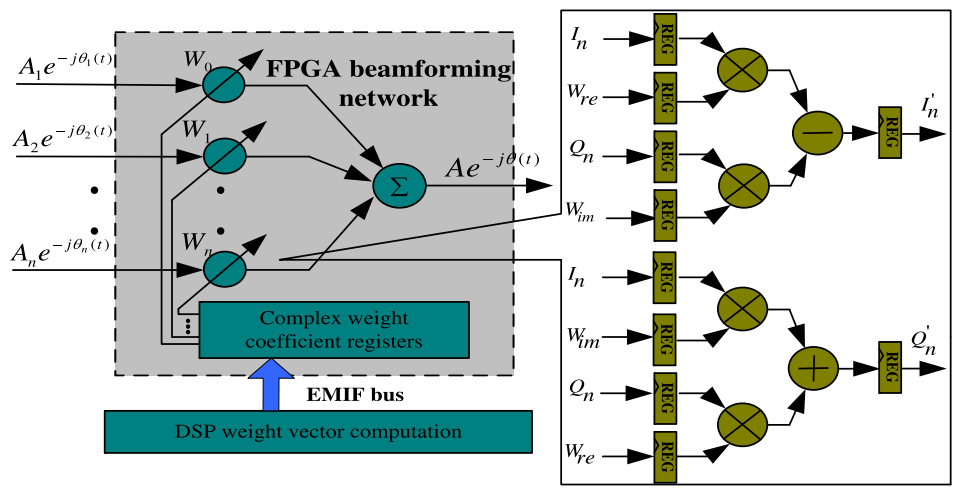

(b) 
(DDCs) and then we get low rate baseband signals. After that, we achieve the DBF algorithm by field programmable gate array (FPGA) and digital signal processor (DSP) and finally different digital beams will be reconstructed by different DUCs to upconvert into analog beam signals.

\subsection{Downconverter module}

We adopt ISL5x16 chip to implement the design. In order to maintain the phase coherence of all channels, all DDCs should be started synchronously after configuration, which plays a crucial role in improving system performance. Fig. 2 (a) shows the synchronization mechanism. To guarantee the synchronization of extern working clock, a no-delay clock driver is used to divide the clock source into four edge-aligned reference clock (REFCLK) signals. To guarantee the synchronization of internal function modules, another no-delay clock driver receives the sync output (SYNCO) signal from master DDC and redistributes it to the sync input (SYNCI) of slave DDCs (as well as feeding it back to the master). As soon as parameter configuration of all devices is complete, DSP will write sync word to the corresponding control register of the master device to make it generate a sync pulse at SYNCO pin. Because all devices receive edge-aligned sync pulses, all internal function modules will return to the same initial state and work synchronously.

\subsection{Beamforming network}

The main function of FPGA includes caching multi-channel data from DDCs and achieving weighted beam summation. Data is sent to FPGA through DDC interface, which will be fed into two internal function modules at the same time. One is cache ram, the other is beamforming network. Data in cache ram will be used for array correction and direction of arrival (DOA) estimation. Beamforming network is the core module in FPGA. Its internal structure is shown in Fig. 2 (b). Complex weight coefficient registers are connected to external bus of DSP. Coefficient is written to corresponding register (REG) asynchronously. Weighting of single channel needs four multiplications and two additions. Using hardware multipliers which are embedded into FPGA can accelerate the computation speed and reduce consumption of logic resources.

\subsection{Weight vector computation}

DSP is used to calculates weight vector $\mathbf{w}$ in formula (10). From the point of view of hardware implementation, eigenvalue decomposition of $R$ matrix is the most complex work. According to formula (4), $R$ is a real symmetric matrix. Considering computational complexity and precision, Jacobi iterative method is adopted [6]. The basic idea of this method is that, through a series of iterations, a orthogonal matrix $Q$ can be found which makes $Q^{T} R Q$ a diagonal matrix. The following is about the problem of solving $\hat{\lambda}$. Assume the matrix $R$ can be decomposed into $U \Lambda U^{H}$, where $U$ represents the eigenvector matrix and $\Lambda$ represents the diagonal matrix composed by the 
eigenvalues of $R$. According to paper [4], $\hat{\lambda}$ meet the following equation:

$$
\sum_{i=1}^{M} \frac{\left|z_{i}\right|^{2}}{\left(\gamma_{i}+\hat{\lambda}\right)^{2}} /\left[\sum_{i=1}^{M} \frac{\left|z_{i}\right|^{2}}{\gamma_{i}+\hat{\lambda}}\right]^{2}=K \quad, \quad \hat{\lambda} \in\left[0, \frac{\gamma_{1}-(M K)^{1 / 2} \gamma_{M}}{(M K)^{1 / 2}-1}\right]
$$

where $\gamma_{i}$ is the diagonal element of $\Lambda$, satisfying $\gamma_{1} \geq \gamma_{2} \geq \cdots \geq \gamma_{M}$ and $z_{i}$ is the element of vector $U^{H} \mathbf{a}\left(\theta_{m}, \phi_{m}\right)$. Paper [7] has proved that the left side of (11) is monotonically decreasing. So we can solve (11) for $\hat{\lambda}$ with bisection method in DSP program. The iteration steps are as follows:

Step 1) Construct function $f(\hat{\lambda})=\sum_{i=1}^{M} \frac{\left|z_{i}\right|^{2}}{\left(\gamma_{i}+\hat{\lambda}\right)^{2}} /\left[\sum_{i=1}^{M} \frac{\left|z_{i}\right|^{2}}{\gamma_{i}+\hat{\lambda}}\right]^{2}-K$. Initialize the boundary interval of $\hat{\lambda}$ as $[a, b]$, where $a=0, b=\left(\gamma_{1}-(M K)^{1 / 2} \gamma_{M}\right) /$ $\left((M K)^{1 / 2}-1\right)$.

Step 2) Calculate $f(a), f(b), f((a+b) / 2)$. For initial values of $a$ and $b$, $f(a) f(b)<0$ must be guaranteed, otherwise the algorithm will not convergence, which means the $K$ value is improperly selected.

Step 3) Set the iteration accuracy of eps. If $f((a+b) / 2)<e p s$, iteration ends and $\hat{\lambda}=(a+b) / 2$. Otherwise, compare $f(a) f((a+b) / 2)$ and $f((a+$ $b) / 2) f(b)$. If $f(a) f((a+b) / 2)<0$, the new boundary interval is $[a,(a+b) / 2]$. Conversely, the new boundary becomes $[(a+b) / 2, b]$. Then, return to the second step.

Step 4) Use $\hat{\lambda}$ and the Equation (10) to get weight vector.

Azimuth information is passed to DSP by direction finding system or host computer to steer beam direction. Although the proposed algorithm is less sensitive to array error, DSP still needs to receive the correction factor of array to guarantee higher accuracy and stability of beam pattern. DSP will multiply the weight vector by the correction factor to get a compensated weight vector as output.

\section{Experimental results}

\subsection{Simulation experiments}

For comparing the performance of $\mathrm{CBF}$ method with the proposed method in theoretical simulation, we choose 5-element uniform circular array with radius of 4 meters as model. Beam patterns in different frequency values are plotted in Fig. 3 (a) and Fig. 3 (b). It can be seen from that, as the frequency decreases, pattern based on conventional beamforming gradually loses directivity. However, pattern based on superdirective beamforming still

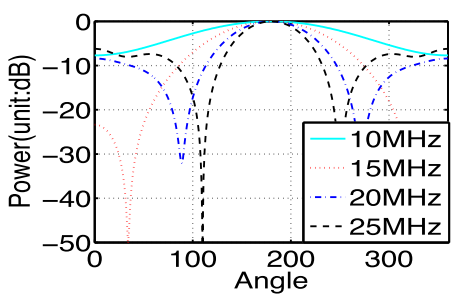

(a)

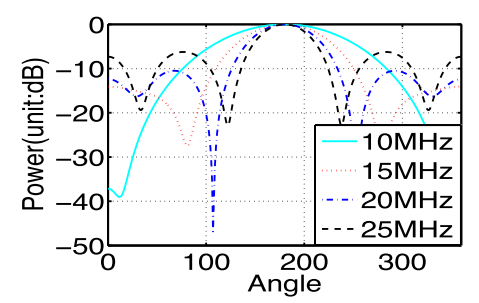

(b) 
keeps a high directive gain. At the high end of frequency, both patterns tend to approximate. Therefore, lower the frequency is, the performance advantage of proposed method over CBF method is more obvious.

\subsection{Hardware experiments}

Based on the description in section 3.3, the selection of $K$ value determines weather the method can convergence, the selection of eps determines the convergence speed. Assuming the eps $=0.0001$, Table. I lists the corresponding convergence time of the algorithm with different number of antenna elements. The CPU clock of TI C6000 floating DSP is $225 \mathrm{MHz}$. FPGA module is implemented with Altera Cyclone IV FPGA chip which consumes 2449 logic elements and 384 9-bit embedded multipliers.

Table I. Convergence time.

\begin{tabular}{|c|c|c|c|c|c|c|}
\hline Antenna elements & 5 & 7 & 9 & 11 & 13 & 15 \\
\hline Time (ms) & 1.19 & 2.77 & 4.69 & 7.2 & 11.1 & 15.58 \\
\hline
\end{tabular}

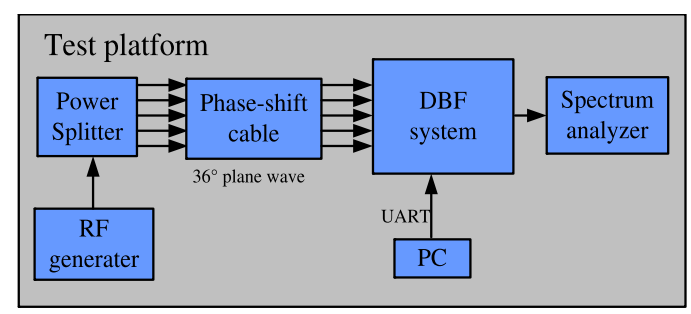

(a)

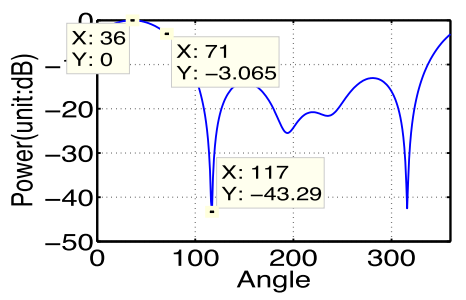

(b)

Fig. 4. (a) Test platform. (b) $13.010 \mathrm{MHz}$ beam pattern $(K=0.6)$.

Fig. 4 (a) is the test platform. Power splitter divides the $13.010 \mathrm{MHZ}$ radio frequency $(\mathrm{RF})$ signal into five signals with equal amplitude and phase offset. Then, phase-shift cables use these signals to simulate a group of planewave signals from $36^{\circ}$ direction. Personal computer (PC) transfers azimuth angle by universal asynchronous serial (UART) to steer the beam direction. Fig. 4 (b) is the actual beam pattern plotted by DSP. The input power of single channel is $-57 \mathrm{dBm}$. Fig. 5 (a), Fig. 5 (b) and Fig. 5 (c) show the summed output power spectrum with the beam pointing towards $36^{\circ}, 71^{\circ}$ and $117^{\circ}$ respectively. These three directions correspond to $0 \mathrm{~dB},-3 \mathrm{~dB}$ and null of beam pattern, as Fig. 4 (b) shows.

\section{Conclusion}

This superdirective digital beamformer is designed for vehicle mounted HF receiving device which uses a 5-elemnet mini array. The design have two advantages. Compared with classic superdirective method, the design can make a good trade-off between robustness and directive gain accordding to actual array error. Therefore, it is more practical in engineering. Compared 


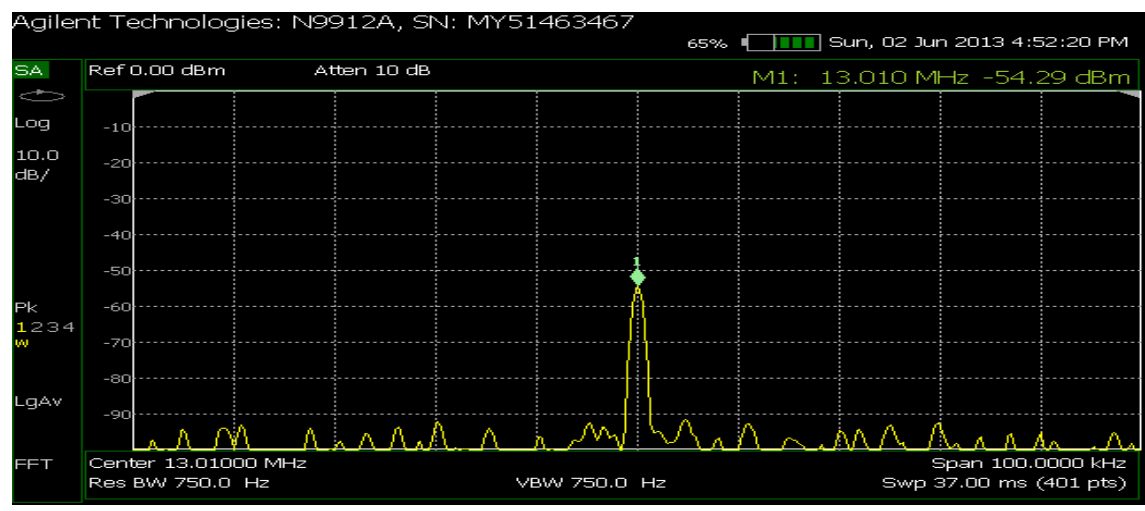

(a)

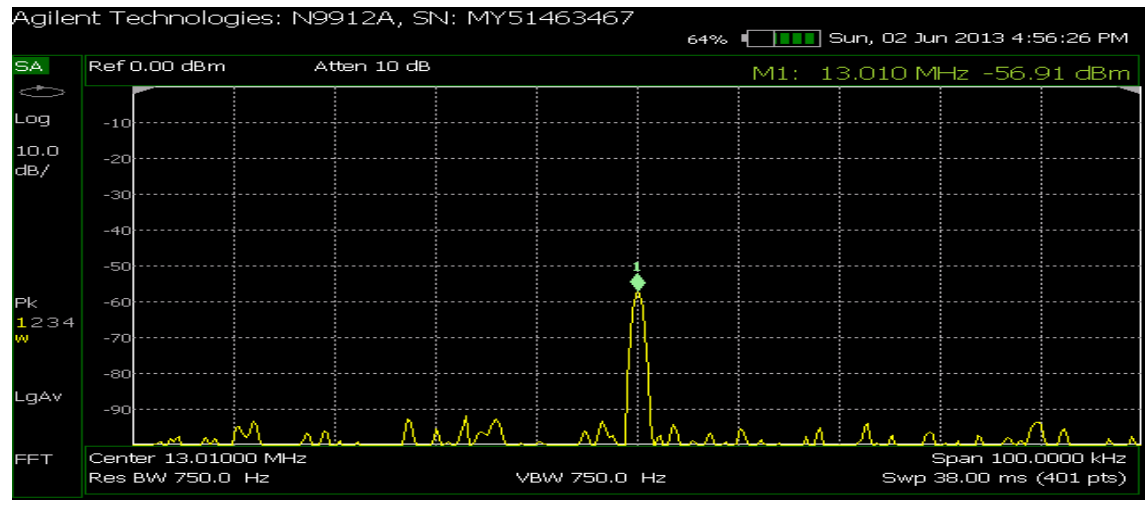

(b)

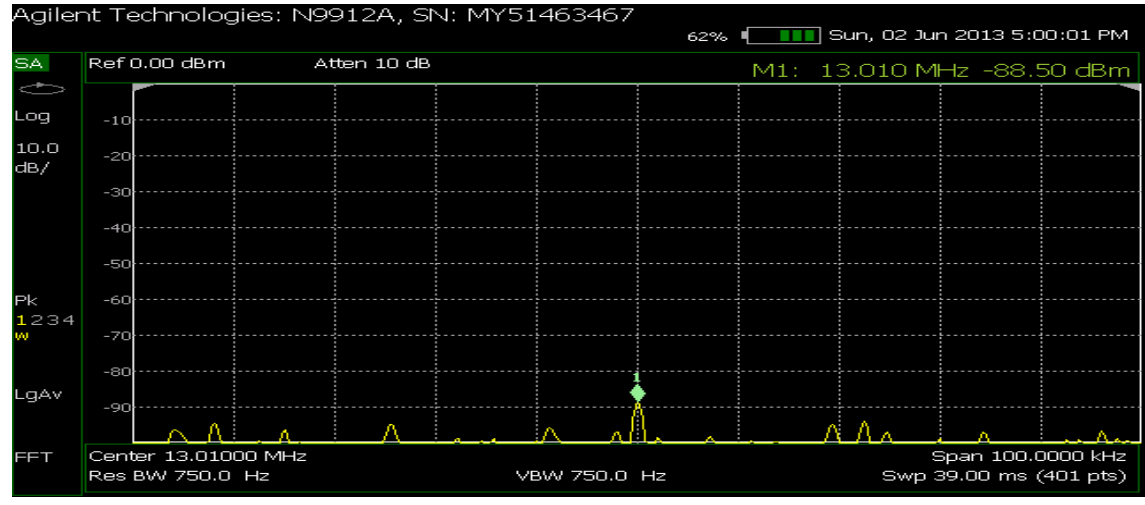

(c)

Fig. 5. (a) Output power of $0 \mathrm{~dB}$ point. (b) Output power of $-3 \mathrm{~dB}$ point.(c) Output power of null.

with conventional beamforming method, its high directive gain increases the anti-jamming ability, especially at low frequency end of HF. Besides, the hardware design concept based on SDR makes it easy to update. Actual experiment results prove the system is feasible and has fast response.

\section{Acknowledgments}

This work is supported by the Fundamental Research Fund for the Central Universities under grant 201221220213 and T201221208. The authors would also like to thank the reviewers for many helpful comments and suggestions, which has enhanced the quality and readability of this paper. 